\title{
Cryopyrin-associated Periodic Syndromes in Italian Patients: Evaluation of the Rate of Somatic NLRP3 Mosaicism and Phenotypic Characterization
}

\author{
Denise Lasigliè, Anna Mensa-Vilaro, Denise Ferrera, Roberta Caorsi, Federica Penco, \\ Giuseppe Santamaria, Marco Di Duca, Giulia Amico, Kenji Nakagawa, Francesca Antonini, \\ Alberto Tommasini, Rita Consolini, Antonella Insalaco, Marco Cattalini, Laura Obici, \\ Romina Gallizzi, Francesca Santarelli, Genny del Zotto, Mariasavina Severino, Anna Rubartelli, \\ Roberto Ravazzolo, Alberto Martini, Isabella Ceccherini, Ryuta Nishikomori, Marco Gattorno, \\ Juan I. Arostegui, and Silvia Borghini
}

\begin{abstract}
Objective. To evaluate the rate of somatic NLRP3 mosaicism in an Italian cohort of mutation-negative patients with cryopyrin-associated periodic syndrome (CAPS).

Methods. The study enrolled 14 patients with a clinical phenotype consistent with CAPS in whom Sanger sequencing of the NLRP3 gene yielded negative results. Patients' DNA were subjected to amplicon-based NLRP3 deep sequencing.

Results. Low-level somatic NLRP3 mosaicism has been detected in 4 patients, 3 affected with chronic infantile neurological cutaneous and articular syndrome and 1 with Muckle-Wells syndrome. Identified nucleotide substitutions encode for 4 different amino acid exchanges, with 2 of them being novel (p.Y563C and p.G564S). In vitro functional studies confirmed the deleterious behavior of the 4 somatic NLRP3 mutations. Among the different neurological manifestations detected, 1 patient displayed mild loss of white matter volume on brain magnetic resonance imaging.

Conclusion. The allele frequency of somatic NLRP3 mutations occurs generally under $15 \%$, considered the threshold of detectability using the Sanger method of DNA sequencing. Consequently, routine genetic diagnostic of CAPS should be currently performed by next-generation techniques ensuring high coverage to identify also low-level mosaicism, whose actual frequency is yet unknown and probably underestimated. (First Release September 15 2017; J Rheumatol 2017;44:1667-73; doi:10.3899/jrheum.170041)
\end{abstract}

Key Indexing Terms:

GENETIC STUDIES

INFLAMMATION

\author{
PEDIATRIC RHEUMATIC DISEASES \\ NEUROLOGIC MANIFESTATIONS
}

\footnotetext{
From these departments of the Istituto Giannina Gaslini, Genoa: Unità Operativa Complessa (UOC) Genetica Medica, UO Pediatria II, Laboratorio Fisiopatologia dell'Uremia, Core facilities, and Neuroradiology Unit; Institute for Maternal and Child Health, Istituto di Ricovero e Cura a Carattere Scientifico (IRCCS) Burlo Garofolo, Trieste; Department of Pediatrics, Immunology and Rheumatology Section, University of Pisa, Pisa; Division of Rheumatology, Department of Pediatric Medicine, Bambino Gesù Children's Hospital, IRCCS, Rome; Pediatric Clinic, Azienda Socio Sanitaria Territoriale (ASST) Spedali Civili and University of Brescia, Brescia; Amyloidosis Research and Treatment Centre, Biotechnology Research Laboratories, Fondazione IRCCS Policlinico San Matteo, Pavia; Department of Pediatrics, Azienda G. Martino, University of Messina, Messina; Department of Pediatrics, Immunology and Rheumatology, University of Torino, Torino; Cell Biology Unit, IRCCS L'Azienda Ospedaliera Universitaria (AOU) San Martino, San Martino, Italy; Department of Immunology, Hospital Clinic-Institut d'Investigacions Biomdiques August Pi i Sunyer (IDIBAPS), Barcelona, Spain; Department of Pediatrics, Graduate School of Medicine, Kyoto University, Kyoto, Japan.

Supported by Giovani Ricercatori-Ricerca Finalizzata grant no. GR-2010-2315933 to SB, and partially funded by the Italian Ministry of Health through both "Cinque per mille" and Ricerca Corrente to the Gaslini Institute, Ricerca Telethon GGP14144 to MG and CERCA
}

\begin{abstract}
Programme/Generalitat de Catalunya (JIA), SAF2015-68472-C2-1-R grant from the Spanish Ministry of Economy and Competitiveness and Fondo Europeo de Desarrollo Regional (JIA) and AC15/00027 grant from the Instituto de Salud Carlos III/Transnational Research Projects on Rare Diseases (JIA).

D. Lasigliè, PhD, UOC Genetica Medica, Istituto Giannina Gaslini; D. Ferrera, PhD, UOC Genetica Medica, Istituto Giannina Gaslini; G. Santamaria, BSc, UOC Genetica Medica, Istituto Giannina Gaslini; G. Amico, MSc, UOC Genetica Medica, Istituto Giannina Gaslini; R. Ravazzolo, MD, UOC Genetica Medica, Istituto Giannina Gaslini; I. Ceccherini, PhD, UOC Genetica Medica, Istituto Giannina Gaslini; S. Borghini, PhD, UOC Genetica Medica, Istituto Giannina Gaslini; M. Di Duca, BSc, Laboratorio Fisiopatologia dell'Uremia, Istituto Giannina Gaslini; F. Penco, PhD, UO Pediatria II Istituto Giannina Gaslini; R. Caorsi, MD, UO Pediatria II Istituto Giannina Gaslini; A. Martini, MD, UO Pediatria II Istituto Giannina Gaslini; M. Gattorno, MD, UO Pediatria II Istituto Giannina Gaslini; A. Mensa-Vilaro, MD, Hospital Clínic-IDIBAPS; J.I. Arostegui, MD, Department of Immunology, Hospital Clínic-IDIBAPS; A. Tommasini, MD, PhD, Institute for Maternal and Child Health, IRCCS Burlo Garofolo; F. Antonini, MSc, Core facilities, Istituto Giannina Gaslini; G. del Zotto, PhD, Core facilities, Istituto Giannina Gaslini; R. Consolini, MD, Department of Pediatrics, Immunology and Rheumatology Section, University of Pisa; A. Insalaco,
\end{abstract}


MD, Division of Rheumatology, Department of Pediatric Medicine, Bambino Gesù Children's Hospital, IRCCS; M. Cattalini, MD, Pediatric Clinic, ASST Spedali Civili and University of Brescia; L. Obici, MD, Amyloidosis Research and Treatment Centre, Biotechnology Research Laboratories, Fondazione IRCCS Policlinico San Matteo; R. Gallizzi, $M D$, Department of Pediatrics, Azienda G. Martino, University of Messina; F. Santarelli, MD, Department of Pediatrics, Immunology and Rheumatology, University of Torino; A. Rubartelli, MD, Cell Biology Unit, IRCSS AOU San Martino; M. Severino, MD, Neuroradiology Unit, Istituto Giannina Gaslini; R. Nishikomori, MD, PhD, Department of Pediatrics, Graduate School of Medicine, Kyoto University; K. Nakagawa, MD, PhD, Department of Pediatrics, Graduate School of Medicine, Kyoto University. Address correspondence to S. Borghini, UOC Genetica Medica, Istituto Giannina Gaslini, Via Gerolamo Gaslini 5, 16148 Genoa, Italy.

E-mail: s.borghini@unige.it

Accepted for publication June 30, 2017.

Cryopyrin-associated periodic syndrome (CAPS) is a group of monogenic autoinflammatory diseases that includes familial cold autoinflammatory syndrome (FCAS), Muckle-Wells syndrome (MWS), and chronic infantile neurological cutaneous and articular syndrome (CINCA). These phenotypes are characterized by chronic or recurrent urticaria-like rash associated with a number of systemic symptoms, and potential structural organ damage. CAPS phenotypes are caused by de novo or dominant mutations of the NLRP 3 gene $^{1}$, which encodes cryopyrin, a key protein of the multiprotein complex called inflammasome that generates the active form of caspase- 1 and interleukin $1 \beta$ (IL-1 $\beta)^{2}$. Among CAPS patients initially described as NLRP3 mutation-negative, next-generation sequencing (NGS) techniques revealed somatic mutations in this gene in 35\% of CINCA cases ${ }^{3,4}$ and $12.5 \%$ of MWS ${ }^{5}$. To date, the clinical phenotype of patients carrying somatic NLRP 3 mosaicism has been indistinguishable from that of patients with germline mutations, with the exception of milder neurological signs $^{3,4,6,7,8}$.

In patients with CINCA, 2 different reports demonstrated that somatic NLRP3 mosaicism occurs at comparable frequency in cells from both myeloid and lymphoid lineages as well as from cells of ectodermic derivation ${ }^{4,8}$. More recently, myeloid-restricted somatic NLRP3 mutations have been described in 2 patients with late-onset but otherwise typical CAPS $^{9,10}$, and in 2 adult patients with the variant-type of Schnitzler syndrome ${ }^{11}$.

In our current study, we have collected Italian patients presenting with the 3 CAPS phenotypes that were NLRP3-negative in studies using the Sanger method of DNA sequencing, with the aim of evaluating the rate of somatic NLRP3 mosaicism and the involved cellular lineages.

\section{MATERIALS AND METHODS}

Patients. The present study enrolled 14 patients affected with clinical symptoms attributable to a CAPS phenotype and NLRP3-negative in genetic studies based on the Sanger method of DNA sequencing. Except for Patient 11 , the patients satisfied diagnostic criteria by both Federici, et al ${ }^{12}$ and Kuemmerle-Deschner, et $a l^{13}$. Patient 11 was included in the study for the presence of repeated episodes of cold-induced fever attacks with elevation of acute-phase reactants. Genomic DNA samples have been extracted from both whole blood and different cellular types by the salting-out method. The study was approved by the Gaslini Ethics Board on May 6, 2004, and was conducted in accordance with the Declaration of Helsinki.

Molecular genetics. For amplicon-based deep sequencing (ADS) studies, amplicons covering all exons of the NLRP3 gene were generated by PCR designed in-house. Library preparation, control quality, and quantification were performed according to manufacturers' instructions. Emulsion PCR was performed on a One Touch2 platform, and sequencing was performed on an Ion Torrent PGM platform using the Ion Torrent PGM HiQ Sequencing kit. The obtained sequences were analyzed using the Torrent Server and the Ion Reporter softwares (Thermo Fischer Scientific Inc.).

Functional analysis of NLRP3 mutations. The functional consequences of the identified NLRP3 variants were evaluated by means of 2 already-described alternative in vitro assays ${ }^{7}$. Briefly, the capability of wild type (wt) and mutant NLRP3 to induce necrosis-like cell death in the THP1 cell line has been quantified by FACS after staining with 7-Amino-actinomycin D. Otherwise, dual-luciferase reporter assay in HEK293FT cells was applied to evaluate induction of nuclear factor- $\kappa \mathrm{B}(\mathrm{NF}-\kappa \mathrm{B})$ activity after transfection with wt and mutant NLRP3 in the presence or absence of its partner ASC (PYCARD).

Purification of immune cell subsets from peripheral blood. Different subpopulations of peripheral blood from patients 1,6 , and 7 were separated by magnetic microbeads $\mathrm{CD} 66 \mathrm{~b}+$ (neutrophils) followed by staining with monoclonal antibodies (mAb) anti-CD19 (B cells), CD3 (T cells), CD16, and CD56 (NK cells), CD14 (monocytes; Beckton Dickinson or Biolegend), and cell sorter technology (FACS ARIA). In Patient 2, CD66b+ neutrophils, CD14+ monocytes, and CD19+/CD3+/CD56+ total lymphocytes were separated from whole blood through cell sorting. Subsequently, B, T, and NK cells were isolated after Ficoll separation of peripheral blood mononuclear cells, staining with above mAb, and sorting.

\section{RESULTS}

The main clinical characteristics of the enrolled patients are summarized in Table 1 and Supplementary Table 1 (available with the online version of this article). ADS revealed somatic NLRP 3 mosaicism in unfractionated peripheral blood in 4 patients (Table 2). The 3 patients with CINCA syndrome (pts 2,6 , and 7) turned out to be carriers of a somatic NLRP3 mosaicism. The amino acid substitutions p.Y563C and p.G564S have never been identified in more than 60,000 human exomes ${ }^{14}$, while the variant p.T433I has been reported as a disease-causing mutation in mosaic status ${ }^{4}$. Interestingly, Patient 2 displayed a clear neurological involvement, with severe and persistent headache, bilateral papilledema, sensorineural deafness, and progressive cognitive impairment. This last condition is to date very rare in the case of somatic mutations ${ }^{4}$ (Table 2). In addition, brain magnetic resonance imaging (MRI), performed on a patient aged 37 years, showed mild loss of white matter volume with enlargement of the lateral ventricles, associated with non-specific periventricular white matter lesions and slightly prominent cerebral sulci (Figure 1).

A somatic NLRP3 mutation was detected in only 1 out of 9 patients with MWS (11.1\%). This patient carried the missense p.R260P amino acid substitution, which has been reported as a disease-causing mutation in germline status ${ }^{15}$. By contrast, neither of the 2 patients with FCAS turned out to be a carrier of a somatic NLRP3 mutation.

To confirm the functional role of the detected somatic

Personal non-commercial use only. The Journal of Rheumatology Copyright @ 2017 . All rights reserved. 
Table 1. Features of patients with CAPS analyzed for NLRP3 mosaicism. All patients are characterized by increment of inflammatory markers at clinical diagnosis. No patients showed AA amyloidosis.

\begin{tabular}{lccccccccccccccc}
\hline Patient No. & 1 & 2 & 3 & 4 & 5 & 6 & 7 & 8 & 9 & 10 & 11 & 12 & 13 \\
\hline Sex & F & M & F & M & F & F & F & M & M & F & M & M & M & F \\
Phenotype & MWS & CINCA & MWS & FCAS & MWS & CINCA & CINCA & MWS & FCAS & MWS & MWS* & MWS & MWS & MWS \\
Birth yr & 2007 & 1971 & 2001 & 1998 & 2000 & 1995 & 1985 & 2001 & 1973 & 1982 & 1986 & 2001 & 1996 & 1976 \\
Age at onset & 2 mos & 2 mos & 10 mos & 2 yrs & 11 yrs & 3 mos & birth & 10.5 yrs & $<1$ yr & 2 yrs & 2 yrs & 5 yrs & 3 yrs & 5 yrs \\
Age at referral & 18 mos & 30 yrs & 5 yrs & 12 yrs & 12 yrs & 15 mos & 8 yrs & 12 yrs & 37 yrs & 31 yrs & 25 yrs & 8 yrs & 17 yrs & 34 yrs \\
Fever & Yes & Yes & Yes & Yes & Yes & Yes & Yes & Yes & Yes & Yes & Yes & Yes & Yes & Yes \\
Urticaria-like rash & Yes & Yes & Yes & Yes & Yes & Yes & Yes & Yes & Yes & Yes & - & Yes & Yes & - \\
Arthritis & - & Yes & Yes & Arthralgias & Yes & Yes & Yes & Yes & - & Yes & - & Yes & Yes & Yes \\
Typical facies & - & Yes & Yes & - & - & - & - & - & - & - & - & - & - & - \\
Headache & - & Yes & - & - & - & Yes & Yes & - & - & - & - & - & - & - \\
Mental retardation & - & Yes & - & - & - & - & - & - & - & - & - & - & - & - \\
Aseptic meningitis & - & Yes & - & - & - & - & - & - & - & - & - & - & - & - \\
Papilloedema & - & Yes & - & - & - & - & Yes & - & - & - & - & - & - \\
Hearing loss & - & Yes & - & - & - & - & Yes & - & Yes & Yes & - & - \\
Bone dysplasia & - & Yes & - & - & - & Yes & Yes & - & - & - & - & - \\
Growth delay & - & Yes & - & Yes & Yes & - & Yes & - & - & - & - & - \\
Uveitis/conjunctivitis & - & Yes & - & - & Yes & - & - & - & - & Yes & - & - \\
Trigger by cold & - & - & - & Yes & - & - & - & - & Yes & - & Yes & - \\
Anti-IL-1 treatment & Yes & Yes & Yes & - & Yes & Yes & Yes & Yes & - & Yes & - & - \\
\hline
\end{tabular}

${ }^{\S}$ Mild (audiometry). * Mild phenotype. CAPS: cryopyrin-associated periodic syndrome; MWS: Muckle-Wells syndrome; CINCA: chronic infantile neurological cutaneous and articular syndrome; FCAS: familial cold autoinflammatory syndrome; IL: interleukin.

Table 2. Frequency of identified somatic mutation of NLRP3. Each frequency value corresponds to the average of 3 independent experiments.

\begin{tabular}{lcccc}
\hline Patient No. & 1 & 2 & 6 & 7 \\
\hline Nucleotide exchange & c.779 G>C & c.1690 G>A & c.1688 A>G & c.1298 C>T \\
Aminoacid substitution & p.R260P & p.G564S & p $563 \mathrm{C}$ & \\
Frequency of mutated allele (\%) in: & & & & \\
Whole blood (first sample) & $10.88(2008)$ & $8.14(2003)$ & 2.71 (unknown) & $5.51(2011)$ \\
Whole blood (second sample) & $9.97(2016)$ & $4.60(2014)$ & $2.17(2016)$ & $5.25(2016)$ \\
B cells & 9.60 & 3.60 & 2.07 & 6.13 \\
NK cells & 9.49 & 6.10 & 1.33 & 8.37 \\
CD14 & 1.23 & 7.10 & 0.79 & 2.87 \\
CD66b & 11.97 & 7.30 & 1.68 & 5.13 \\
CD3 & 14.70 & 3.20 & 4.93 & 7.23 \\
Saliva (\% CD45 cells) & $11.03(57.5)$ & $4.70(32.2)$ & $1.87(56.1)$ & $1.73(59.1)$ \\
Urine & 8.53 & 0 & 2.97 & NA \\
Swab & NA & 6.70 & 4.27 & 0.60 \\
Hair & NA & 0 & NA & NA \\
Nail & NA & 7.80 & NA & NA \\
\hline
\end{tabular}

Round brackets: year of blood sampling. NA: not analyzed; NK: natural killer.

mutations, we have taken advantage of 2 different in vitro assays. Compared to wt cryopyrin, mutant forms of protein rapidly induce necrotic cell death when transiently transfected in human monocytic THP-1 cells (Figure 2A). In addition, co-expression of mutant cryopyrin with its partner ASC in HEK293FT cells causes enhanced NF- $\kappa B$ reporter activity (Figure 2B). Notably in both analyses, the effect of the newly identified mutations appears to be stronger than that of the known disease-causing mutations p.R260W and p.Y570C.
As previously reported, the frequency of the mutated $N L R P 3$ allele remained similar in blood samples obtained over several years ${ }^{5}$.

In some patients, the functional analysis of IL-1 secretion was performed with variable results. Patients 6 and 7 displayed a high IL-1 secretion after lipopolysaccharide (LPS) stimulation similar to that observed in patients with CAPS carrying germline mutations (data not shown). Patient 2 displayed a slight increase of IL-1 secretion after LPS stimulation with the same accelerated pattern observed in

Personal non-commercial use only. The Journal of Rheumatology Copyright $\odot$ 2017. All rights reserved. 


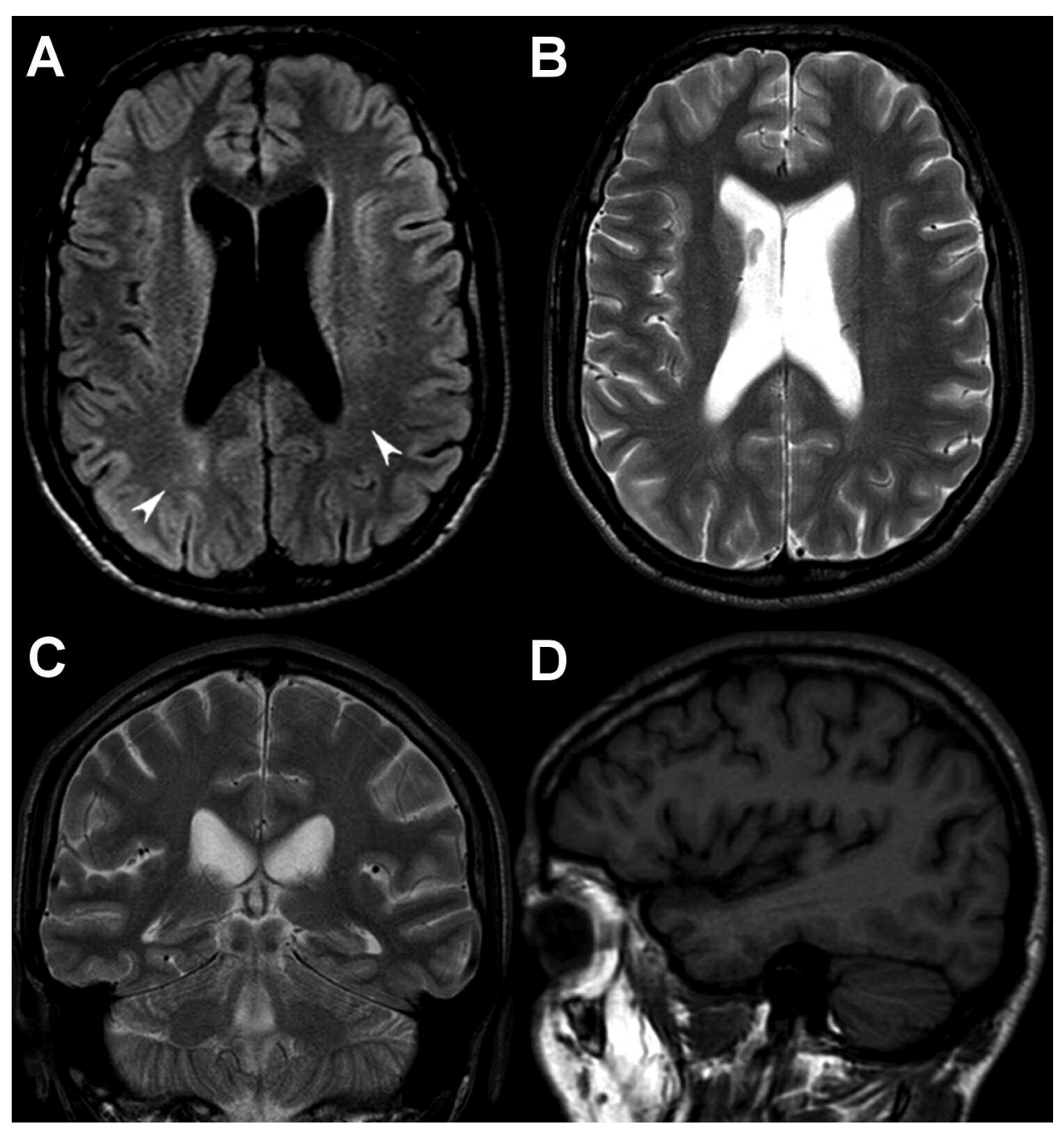

Figure 1. Brain magnetic resonance imaging performed on a 37-year-old patient. Axial fluid attenuated inversion recovery (A) and T2-weighted (B) images reveal mild loss of white matter volume with secondary ventricular enlargement. A few nonspecific hyperintense lesions are noted in the periventricular regions (arrows). Coronal T2-weighted (C) and sagittal T1-weighted (D) images reveal slightly prominent cerebral sulci.

CAPS $^{16}$ (Supplementary Figure 1, available with the online version of this article).

In all patients bearing somatic NLRP3 mosaicism, we have analyzed the presence of the mutant allele in different tissues. All blood cell subpopulations as well as the epithelial cells displayed the mutant allele. Apart from a few exceptions, no significant differences in the frequency of mutant allele were observed. In particular, 2 of 5 ectodermic tissues (epithelial cells from urine and hair bulb) were negative for mutant allele in Patient 2, and monocytes displayed a very low frequency in Patient 1.

\section{DISCUSSION}

In recent years, a number of studies have demonstrated that somatic NLRP3 mutations are responsible for CAPS phenotypes in a relevant percentage of patients who were negative in previous studies using conventional Sanger sequencing.
Actually, this phenomenon accounts for $35 \%$ of CINCA and $12.5 \%$ of MWS patients ${ }^{3,4,5}$. To date, no evidence of somatic NLRP3 mosaicism has been reported in FCAS. Our results in Italian patients confirm that somatic NLRP3 mosaicism is a genetic mechanism underlying CINCA and MWS phenotypes.

In line with published data, $50 \%$ of the NLRP 3 mutations detected in this work are novel, and one of the remaining variants had been described only in mosaic status ${ }^{4}$. This observation enforces the hypothesis that these mutations in germline status could be incompatible with life. Actually, in vitro functional studies clearly showed their gain-of-function effect, even stronger for mutations found in mosaic status compared to germline mutations.

To date, the patients carrying somatic NLRP3 mosaicism did not present clear clinical differences when compared to patients carrying germline mutations, with the exception of milder neurological signs in patients with mosaicism ${ }^{4}$. To our

Personal non-commercial use only. The Journal of Rheumatology Copyright (C) 2017. All rights reserved. 
A.

\section{THP-1 cell death}

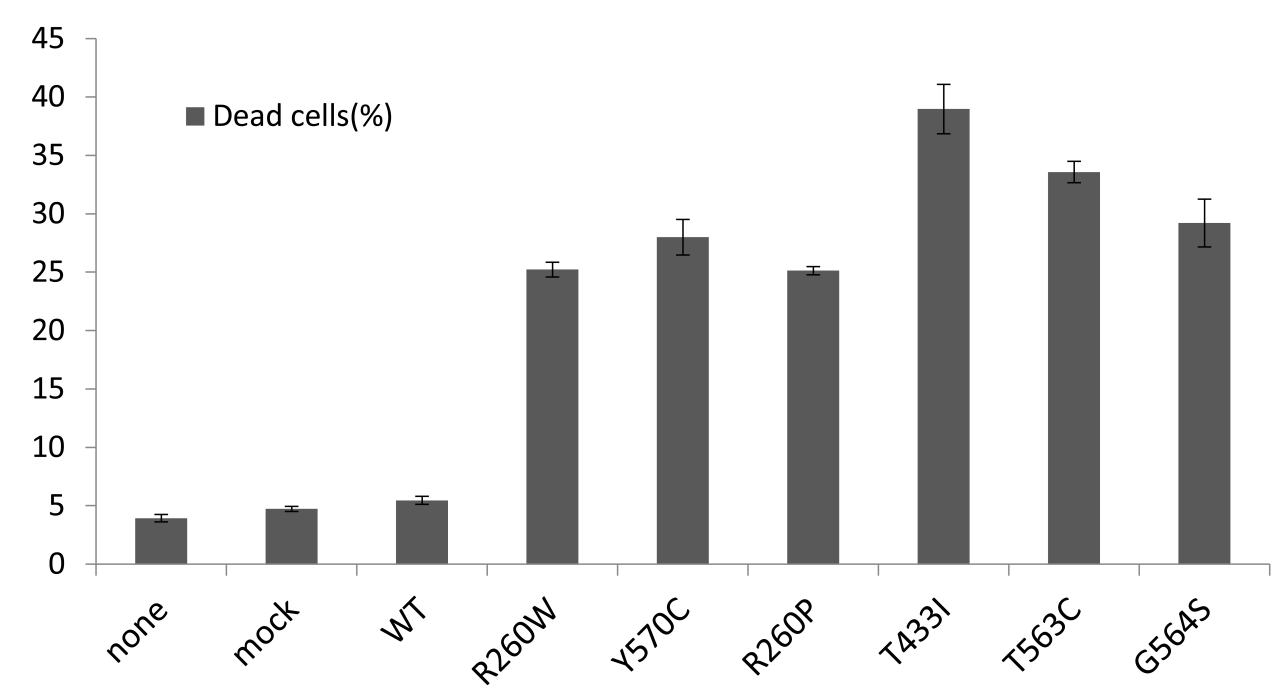

B.

$14 \quad$ NF-kB activation

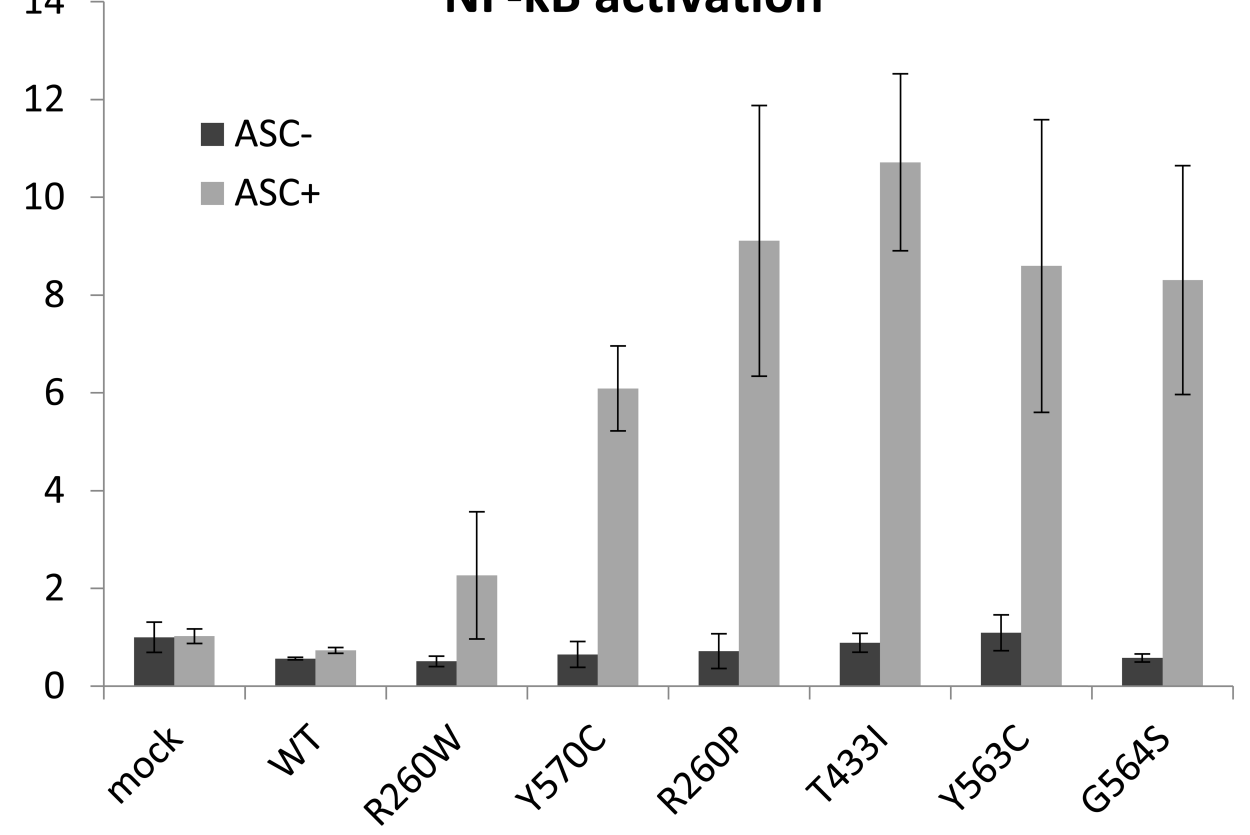

Figure 2. Functional analysis of NLRP3 mutations. (A) Mutated NLRP3 proteins induce cell death in transfected THP-1 cells. Bars represent percentages of dead cells (7-Amino-actinomycin D-positive) among the NLRP3-GFP+ cells as means of triplicate experiments. Error bars correspond to SD. Constructs with NLRP3-GFP-bearing p.Arg260Trp and p.Tyr570Cys (R260W and Y260C) are used as positive controls. Activity of wt NLRP3-GFP is represented. None $=$ not transfected; mock $=$ cells subjected to transfection protocol without vectors. (B) Mutated NLRP3 induce NF-KB activation in transfected HEK293FT cells when ASC is cotransfected to reconstitute an active inflammasome. NF- $\mathrm{KB}$ activation is shown as fold-change compared to the activity of empty control vector. Bars represent means of triplicate experiments and error bars correspond to SD. ASC+ = transfection of NLRP3 expression vector together with ASC expression vector; $\mathrm{ASC}-=$ transfection of NLRP3 expression vector with empty expression vector. WT: wild type; NF- $\mathrm{KB}$ : nuclear factor- $\mathrm{\kappa B}$.

knowledge, Patient 2 represents the first patient with CINCA carrying a somatic NLRP3 mutation with minor structural brain abnormalities on MRI, including reduction of white matter volume and prominent cerebral sulci. The amino acid exchange he carries (p.G564S) is novel, suggesting that this neurological implication could be a peculiar phenotypic

Personal non-commercial use only. The Journal of Rheumatology Copyright @ 2017 . All rights reserved. 
effect, or alternatively could be related to the specific tissue distribution of the mutant allele, which is not easily analyzable.

Similar considerations could also explain a severe phenotype in case of very low mosaicism frequency, as in Patient 6 (2.7\% of mutated allele), characterized by early onset, arthritis, and bone dysplasia. Notably, because a given frequency of the mutated allele corresponds to a double frequency of heterozygous cells, the presence of an active NLRP 3 mutation in only a portion of about $5.4 \%$ of circulating leukocytes appears to be sufficient to cause the clinical manifestations of CINCA syndrome. But a lower frequency (2.4\%) of a different mutation (p.T348M) was not sufficient to induce the clinical manifestations in the asymptomatic mother of a patient with MWS ${ }^{17}$, thus confirming that not only the amount of mutant allele but also the characteristic of the mutation and likely its tissue distribution are going to determine the phenotypic effects of an NLRP3 mosaicism.

The patients here detected as carrying somatic NLRP3 mosaicism display the variant allele in tissues derived from different embryonic layers, suggesting that the somatic mutational event occurred very early during the ontogenesis.

Consistent with most of the patients already described $^{3,4,5,7}$, the level of mosaicism in our cases is below $15 \%$, commonly considered the threshold of detectability using conventional Sanger sequencing. In contrast with some other countries, in Italy the lack of a positive genetic test at standard Sanger analysis does not prevent a CAPS diagnosis in patients with a clear phenotype. This leads to the use of anti-IL-1 treatments, as experienced by our patients, with the same excellent results observed in patients with germline mutation ${ }^{18}$. However, a correct genetic diagnosis is essential to allow the patient to make informed choices about his/her reproductive future. Though the somatic mosaicism rate may differ in tissues and it is not usually determined in gonadal tissues, the risk of transmission of the mutated allele to the offspring is regarded as likely low, and the genetic counseling is quite challenging. Indeed, there is evidence that somatic NLRP3 mutations also affect germline cells, with consequent vertical transmission ${ }^{17}$

Detection of mosaicism in autoinflammatory disease is increasing, having recently been demonstrated also in the TMEM173, NOD2, TNFRSF1A, and NLRC4 genes ${ }^{19,20,21,22}$. To date, among CAPS-like phenotypes, genetic heterogeneity has been found in a small proportion of FCAS cases ${ }^{23,24}$ and in a single patient with CINCA $^{22}$, while an approach based on whole exome sequencing to search for a novel candidate gene was able to identify only a mutation of the NLRP 3 gene in mosaic status ${ }^{25}$. For this reason, in the case of clinical features attributable to CAPS, we strongly support the need to replace Sanger sequencing with NGS techniques that ensure high coverage to identify low-level mosaicism, whose actual frequency is still unknown and likely underestimated.

Our work confirms the presence of somatic NLRP3 mosaicisms in Italian patients with CAPS who were negative for germline mutations with conventional Sanger analysis. This evidence highlights the need to update the routine molecular diagnosis for CAPS by means of high-coverage NGS, which can also identify low-level mosaicism in the NLRP3 gene.

\section{ACKNOWLEDGMENT}

We are extremely grateful to all the families who have participated in this study, and to Loredana Velo and Laura Carenini for excellent secretarial assistance.

\section{ONLINE SUPPLEMENT}

Supplementary material accompanies the online version of this article.

\section{REFERENCES}

1. Hoffman H, Mueller J, Broide D, Wanderer A, Kolodner R. Mutation of a new gene encoding a putative pyrin-like protein causes familial cold autoinflammatory syndrome and Muckle-Wells syndrome. Nat Genet 2001;29:301-5.

2. Martinon F, Burns K, Tschopp J. The inflammasome: a molecular platform triggering activation of inflammatory caspases and processing of proIL-beta. Mol Cell 2002;10:417-26.

3. Izawa K, Hijikata A, Tanaka N, Kawai T, Saito MK, Goldbach-Mansky R, et al. Detection of base substitution-type somatic mosaicism of the NLRP3 gene with $>99.9 \%$ statistical confidence by massively parallel sequencing. DNA Res 2012;19:143-52.

4. Tanaka N, Izawa K, Saito MK, Sakuma M, Oshima K, Ohara O, et al. High incidence of NLRP3 somatic mosaicism in patients with chronic infantile neurologic, cutaneous, articular syndrome: results of an International Multicenter Collaborative Study. Arthritis Rheum 2011;63:3625-32.

5. Nakagawa K, Gonzalez-Roca E, Souto A, Kawai T, Umebayashi H, Campistol JM, et al. Somatic NLRP3 mosaicism in Muckle-Wells syndrome. A genetic mechanism shared by different phenotypes of cryopyrin-associated periodic syndromes. Ann Rheum Dis 2015;74:603-10.

6. Saito M, Fujisawa A, Nishikomori R, Kambe N, Nakata-Hizume M, Yoshimoto M, et al. Somatic mosaicism of CIAS1 in a patient with chronic infantile neurologic, cutaneous, articular syndrome. Arthritis Rheum 2005;52:3579-85.

7. Saito M, Nishikomori R, Kambe N, Fujisawa A, Tanizaki H, Takeichi K, et al. Disease-associated CIAS1 mutations induce monocyte death, revealing low-level mosaicism in mutation-negative cryopyrin-associated periodic syndrome patients. Blood 2008;111:2132-41.

8. Aróstegui JI, Lopez Saldaña MD, Pascal M, Clemente D, Aymerich M, Balaguer F, et al. A somatic NLRP3 mutation as a cause of a sporadic case of chronic infantile neurologic, cutaneous, articular syndrome/neonatal-onset multisystem inflammatory disease: novel evidence of the role of low-level mosaicism as the pathophysiologic mechanism underlying mendelian inherited diseases. Arthritis Rheum 2010;62:1158-66.

9. Mensa-Vilaro A, Teresa Bosque M, Magri G, Honda Y, Martínez-Banaclocha H, Casorran-Berges M, et al. Late onset cryopyrin-associated periodic syndrome due to myeloid-restricted somatic NLRP3 mosaicism. Arthritis Rheumatol 2016;68:3035-41.

10. Zhou Q, Aksentijevich I, Wood GM, Walts AD, Hoffmann P, Remmers EF, et al. Brief report: cryopyrin-associated periodic syndrome caused by a myeloid-restricted somatic NLRP3 mutation. Arthritis Rheumatol 2015;67:2482-6.

Personal non-commercial use only. The Journal of Rheumatology Copyright @ 2017 . All rights reserved. 
11. de Koning HD, van Gijn ME, Stoffels M, Jongekrijg J, Zeeuwen PL, Elferink MG, et al. Myeloid lineage-restricted somatic mosaicism of NLRP3 mutations in patients with variant Schnitzler syndrome. J Allergy Clin Immunol 2015;135:561-4.

12. Federici S, Sormani MP, Ozen S, Lachmann HJ, Amaryan G, Woo P, et al. Evidence-based provisional clinical classification criteria for autoinflammatory periodic fevers. Ann Rheum Dis 2015; 74:799-805

13. Kuemmerle-Deschner JB, Ozen S, Tyrrell PN, Kone-Paut I, Goldbach-Mansky R, Lachmann H, et al. Diagnostic criteria for cryopyrin-associated periodic syndrome (CAPS). Ann Rheum Dis 2017;76:942-7.

14. Lek M, Karczewski KJ, Minikel EV, Samocha KE, Banks E, Fennell T, et al. Analysis of protein-coding genetic variation in 60,706 humans. Nature 2016;536:285-91.

15. Neven B, Callebaut I, Prieur AM, Feldmann J, Bodemer C, Lepore $\mathrm{L}$, et al. Molecular basis of the spectral expression of CIAS1 mutations associated with phagocytic cell-mediated autoinflammatory disorders CINCA/NOMID, MWS, and FCU. Blood 2004;103:2809-15.

16. Tassi S, Carta S, Delfino L, Caorsi R, Martini A, Gattorno M, et al. Altered redox state of monocytes from cryopyrin-associated periodic syndromes causes accelerated IL-1beta secretion. Proc Natl Acad Sci U S A 2010;107:9789-94.

17. Jiménez-Treviño S, González-Roca E, Ruiz-Ortiz E, Yagüe J, Ramos E, Aróstegui JI. First report of vertical transmission of a somatic NLRP3 mutation in cryopyrin-associated periodic syndromes. Ann Rheum Dis 2013;72:1109-10.

18. Lepore L, Paloni G, Caorsi R, Alessio M, Rigante D, Ruperto N, et al. Follow-up and quality of life of patients with cryopyrin-associated periodic syndromes treated with Anakinra. J Pediatr 2010;157:310-5.e1.
19. de Inocencio J, Mensa-Vilaro A, Tejada-Palacios P, E nriquez-Merayo E, González-Roca E, Magri G, et al. Somatic NOD2 mosaicism in Blau syndrome. J Allergy Clin Immunol 2015;136:484-7.e2.

20. Rowczenio DM, Trojer H, Omoyinmi E, Aróstegui JI, Arakelov G, Mensa-Vilaro A, et al. Brief report: association of tumor necrosis factor receptor-associated periodic syndrome with gonosomal mosaicism of a novel 24-nucleotide TNFRSF1A deletion. Arthritis Rheumatol 2016;68:2044-9.

21. Liu Y, Jesus AA, Marrero B, Yang D, Ramsey SE, Montealegre Sanchez GA, et al. Activated STING in a vascular and pulmonary syndrome. N Engl J Med 2014;371:507-18.

22. Kawasaki Y, Oda H, Ito J, Niwa A, Tanaka T, Hijikata A, et al. Identification of high frequency somatic NLRC4 mutation as a cause of autoinflammation by pluripotent cell-based phenotypic dissection. Arthritis Rheumatol 2017;69:447-59.

23. Borghini S, Tassi S, Chiesa S, Caroli F, Carta S, Caorsi R, et al. Clinical presentation and pathogenesis of cold-induced autoinflammatory disease in a family with recurrence of a NLRP12 mutation. Arthritis Rheum 2011;63:830-9.

24. Jéru I, Duquesnoy P, Fernandes-Alnemri T, Cochet E, Yu J, Lackmy-Port-Lis M, et al. Mutations in NALP12 cause hereditary periodic fever syndromes. Proc Natl Acad Sci U S A 2008;105:1614-9.

25. Omoyinmi E, Melo Gomes S, Standing A, Rowczenio DM, Eleftheriou D, Klein N, et al. Brief report: whole-exome sequencing revealing somatic NLRP3 mosaicism in a patient with chronic infantile neurologic, cutaneous, articular syndrome. Arthritis Rheumatol 2014;66:197-202. 